\title{
Chemical composition of the Taurus-Auriga association ${ }^{\star}$
}

\author{
V. D’Orazi ${ }^{1}$, K. Biazzo ${ }^{2}$, and S. Randich ${ }^{2}$ \\ ${ }^{1}$ INAF - Osservatorio Astronomico di Padova, Vicolo dell'Osservatorio 5, 3522 Padova, Italy \\ e-mail: valentina.dorazi@oapd.inaf.it \\ 2 INAF - Osservatorio Astrofisico di Arcetri, Largo E. Fermi 5, 50125 Firenze, Italy
}

Received 19 August 2010 / Accepted 17 November 2010

\begin{abstract}
Context. The Taurus-Auriga association is perhaps the most famous prototype of a low-mass star forming region, surveyed at almost all wavelengths. Unfortunately, like several other young clusters/associations, this $\mathrm{T}$ association lacks an extensive abundance analysis determination.

Aims. We present a high-resolution spectroscopic study of seven low-mass members of Taurus-Auriga, including both weak-lined and classical T Tauri stars designed to help robustly determine their metallicity.

Methods. After correcting for spectral veiling, we performed equivalent width and spectral synthesis analyses using the GAIA set of model atmospheres and the 2002 version of the code MOOG.

Results. We find a solar metallicity, obtaining a mean value of $[\mathrm{Fe} / \mathrm{H}]=-0.01 \pm 0.05$. The $\alpha$-element $\mathrm{Si}$ and the Fe-peak one $\mathrm{Ni}$ confirm a solar composition. Our work shows that the dispersion among members is well within the observational errors at variance with previous claims. As in other star forming regions, no metal-rich members are found, reinforcing the idea that old planet-host stars form in the inner part of the Galactic disc and subsequently migrate.
\end{abstract}

Key words. stars: abundances - open clusters and associations: individual: Taurus-Auriga

\section{Introduction}

The Taurus-Auriga (Tau-Aur) association is the nearest large star-forming region (SFR), with a close distance of $d=140 \mathrm{pc}$ (e.g., Kenyon et al. 1994). Given its lack of massive O/B stars, this $\mathrm{T}$ association in the past 50 years has become a standard region to study the low-mass star formation processes. Tau-Aur is widely spread across the Northern sky ( 100 square degrees, Scelsi et al. 2007a) and contains hundreds of low-mass members; Rebull et al. (2010) found 148 new candidate members, of which 34 were confirmed by spectroscopic follow-up. The bulk of stars presents an average age of $\sim 1$ Myr (e.g., Briceño et al. 1999).

The whole region has been observed at almost all wavelengths, from infrared to X-rays (e.g., Itoh et al. 1996; Briceño et al. 1999; Davis et al. 2008; Luhman et al. 2010). Kenyon \& Hartmann (1995, hereafter KH95) provided a thorough investigation of the stellar population, from class 0 (proto-stars) to class II/III (i.e. classical and weak-lined T Tauri stars, respectively - hereafter CTTs and WTTs). They analysed optical and infrared photometric observations and presented colourmagnitude and colour-colour diagrams, luminosity and mass functions, along with information on the near-infrared excess and accretion properties. Lithium abundance studies of this association have been also performed over the past decade, such as, e.g., Basri et al. (1991), Magazzú et al. (1992), Martìn et al. (1994), and Sestito et al. (2008), who presented a (re)assessment of Li I abundances in WTTs and CTTs. Güdel et al. 2007 carried out an extensive X-ray survey (XMM-Newton Extended Survey of the Taurus Molecular Cloud-XEST) covering an area

\footnotetext{
^ Based on observations collected at Telescopio Nazionale Galileo, Canary Islands (Spain). Program AOT16/07B.
}

of $\sim 5$ square degrees and concentrating mainly on the higher stellar density regions.

Whereas all these studies have helped to characterise the stellar population, Tau-Aur shares with other nearby SFRs the lack of an accurate abundance analysis.

The determination of chemical composition of SFRs is instead critically important to a variety of astrophysical issues, in both planetary and stellar contexts, as we previously discussed in our pilot project focusing on the Orion association (D'Orazi et al. 2009, hereafter D09). At variance with OB associations, whose abundances can provide an independent test of the socalled triggered star formation scenario (e.g., Blaauw 1991) and indicate whether there is local enrichment, we would expect to measure a very homogeneous composition for $\mathrm{T}$ associations.

As is well known, giant planets are preferentially found around metal-rich main sequence stars (Santos et al. 2004, and reference therein). The natural question hence arises as to whether in the first epochs of planetary formation (discs of $\mathrm{T}$ Tauri stars are commonly accepted as planet birthplaces) the probability of hosting a giant planet depends on the star's metallicity.

On the other hand, and very interestingly, several studies (e.g., Luhman 2004) have found significant differences between the initial mass function (IMF) derived for the Tau-Aur and those derived for denser systems containing massive members, e.g., the Trapezium, with the former containing a too small number of brown dwarfs according to the standard IMF (Briceño et al. 2002) and a surplus of late-K and early-M stars (Luhman et al. 2009). These differences can be attributed to different conditions of the environment where the stars were formed. Some studies have claimed that the IMF slope may depend on metallicity, in the sense that metal-rich environments tend to produce more 
Table 1. Log of observations and stellar properties.

\begin{tabular}{|c|c|c|c|c|c|c|c|c|}
\hline Star & $\begin{array}{c}\text { Obs. date } \\
\text { (yy-mm-dd) }\end{array}$ & $\begin{array}{l}\text { Exp. time } \\
\text { (s) }\end{array}$ & $S / N_{6708 \AA}$ & $\begin{array}{c}V \\
(\mathrm{mag})\end{array}$ & Sp. type & $\begin{array}{l}T_{\text {eff }} \\
(\mathrm{K})\end{array}$ & Class & $\begin{array}{r}v \sin i_{\text {lit. }} \\
\left(\mathrm{km} \mathrm{s}^{-1}\right)\end{array}$ \\
\hline GM Aur & 2007-09-08 & 3600 & 110 & 12.03 & K7 & 4060 & II & 12.4 \\
\hline BP Тau & $2007-12-20$ & $\begin{array}{l}3600 \\
1168\end{array}$ & 110 & 12.16 & K7 & 4060 & II & 7.8 \\
\hline V826 Tau A & 2007-09-19 & 3600 & 90 & 12.11 & K7 & 4060 & III & 4.2 \\
\hline Hubble 4 & 2007-09-20 & $\begin{array}{c}2 \times 3000 \\
2150\end{array}$ & 120 & 12.67 & K7 & 4060 & III & 12.8 \\
\hline RX J0438.2+2023 & $2007-10-30$ & $\begin{array}{l}3600 \\
2550\end{array}$ & 110 & 12.22 & K2 & 4900 & III & 8.0 \\
\hline RX J0423.7+1537 & 2007-09-19 & 2200 & 140 & 11.29 & K2 & 4900 & III & $\ldots$ \\
\hline LkCa 19 & $2007-12-20$ & 1270 & 100 & 10.85 & K0 & 5250 & III & 18.6 \\
\hline
\end{tabular}

low-mass stars $\left(M<0.7 M_{\odot}\right)$ than metal-poor systems (Larson 2005; Da Rio et al. 2009, and references therein). The obvious question is: could metallicity play a role in the lower fraction of very low-mass stars and brown dwarfs detected in Taurus with respect to Trapezium (Briceño et al. 2002)? More generally, we may ask whether there is a difference between chemical compositions of low-mass and high-mass SFRs or, whether, SFRs represent a chemically homogeneous class of objects?

Available information on Tau-Aur abundances comes from the previous works by Padgett (1996) and Santos et al. (2008). First, Padgett (1996) analysed eight WTT members and derived a solar composition, i.e. an average value of $[\mathrm{Fe} / \mathrm{H}]=$ $0.01 \pm 0.13$ (rms), with a significant scatter among members ranging from -0.11 to +0.22 . Santos et al. (2008) presented the iron abundances of three Taurus members: in this case, the spread in metallicity is also large, varying from -0.18 to +0.05 with a mean value of $[\mathrm{Fe} / \mathrm{H}]=-0.07 \pm 0.12(\mathrm{rms})$. Given the quite large star-to-star variation obtained by both studies, a new accurate abundance analysis for this association is warranted, to minimize the impact of the observational uncertainties and ascertain whether the metallicity scatter among members is indeed real or not.

In a different framework, Scelsi et al. (2007b) obtained coronal abundances for a sample of X-ray bright members of Taurus, finding that the iron abundance in the corona is significantly (by a factor of $\sim 5$ ) lower than the solar photospheric value. More generally, their coronal abundances revealed a pattern in agreement with those obtained in previous studies. Specifically, $\mathrm{X}$-ray observations of young and/or active stars have unveiled a so-called FIP (first ionization potential)-dependent abundance trend: low FIP elements (FIP $\leq 10 \mathrm{eV}$, like iron, silicon, nickel) are under-abundant in the corona with respect to the photospheric values (the so-called inverse-FIP effect, for further details see Brinckman et al. 2001; Audard et al. 2003; Scelsi et al. 2007b). As stressed by Scelsi et al., however, this FIP-related pattern for Tau-Aur, as well as for other young stars, was obtained by comparing the coronal abundances to the solar photospheric values, rather than the stellar photospheric abundances. Different or no FIP effects were indeed found when considering the correct stellar photospheric abundances (e.g., Sanz-Forcada et al. 2004; Maggio et al. 2007). Determining the photospheric abundances of Tau-Aur members with measured coronal abundances thus appears critical to confirm whether a FIP-related effect is present in these stars or not.

In this work, we present a high-resolution spectroscopic study based on seven low-mass members of the Tau-Aur association. The sample was selected specifically to determine accurate abundances, by discarding, e.g., fast rotators, strong accretors, and/or binary stars. Most importantly, this is one of the first sample that also includes $\mathrm{T}$ Tauri stars that have not yet dissipated their circumstellar discs and reveal moderate accretion from the surrounding material. Details of the observations and data reduction are given in Sect. 2; Sect. 3 describes the estimate of and correction for spectral veiling, along with the abundance analysis procedure. The results and the scientific implications are illustrated in Sects. 4 and 5, while in Sect. 6 a conclusive summary is given.

\section{Observations and data reduction}

Our sample consists of seven low-mass members of the Tau-Aur association, of which two are CTTs and five WTT stars, respectively. The sample was selected from the large database provided in XEST by Güdel et al. (2007).

Single K-type members were chosen with projected rotational velocities $(v \sin i)$ not larger than $\sim 30 \mathrm{~km} \mathrm{~s}^{-1}$ and a mass accretion rate below $\log \dot{M}=-7.5 M_{\odot} \mathrm{yr}^{-1}$. For stars rotating faster than $30 \mathrm{~km} \mathrm{~s}^{-1}$ the abundance analysis becomes infeasible, because of rotational blending. The upper limit to the accretion rate allowed by including in the sample also CTTs and, at the same time, discarding extremely strong accretors, for which abundance analysis is no longer possible. Basic properties of our sample are given in Table 1, where we list the star name, the observation date, and the exposure times in Cols. 1-3, respectively; signal-to-noise ratio $(S / N)$ per pixel measured around the $\mathrm{Li}$ I line at $6708 \AA$ in the co-added spectra are reported in Col. 4. The $V$ magnitudes and spectral-types are listed in Cols. 5 and 6, respectively, while the initial effective temperature $\left(T_{\text {eff }}\right)$ from Güdel et al. (2007) is given in Col 7. Finally, in Col. 8 we show the classification as CTT (Class II) or WTT (Class III) stars, while projected rotational velocities from XEST catalogue are in Col 9.

The observations were carried out in service mode at the Telescopio Nazionale Galileo (Roque de los Muchachos, Canary Islands) with the high-resolution spectrograph SARG, during seven nights of observations between August and December 2007. We used the $61 \AA \mathrm{mm}^{-1} \mathrm{CD} 3$ yellow grism $\left(\lambda_{\text {blaze }}=5890 \AA\right)$, which resulted in a spectral coverage between $4620 \AA$ and $7920 \AA$, along with a $1.6^{\prime \prime}$ slit, providing a resolution $R \sim 29000$. As we discuss below, we also acquired a solar spectrum with the same instrumental set-up, which permits us to carry out a differential abundance analysis (see Sect. 3) with respect to the Sun. Data reduction was performed within the ECHELLE context in MIDAS following the standard procedures of bias subtraction, flat-field correction, order definition and 


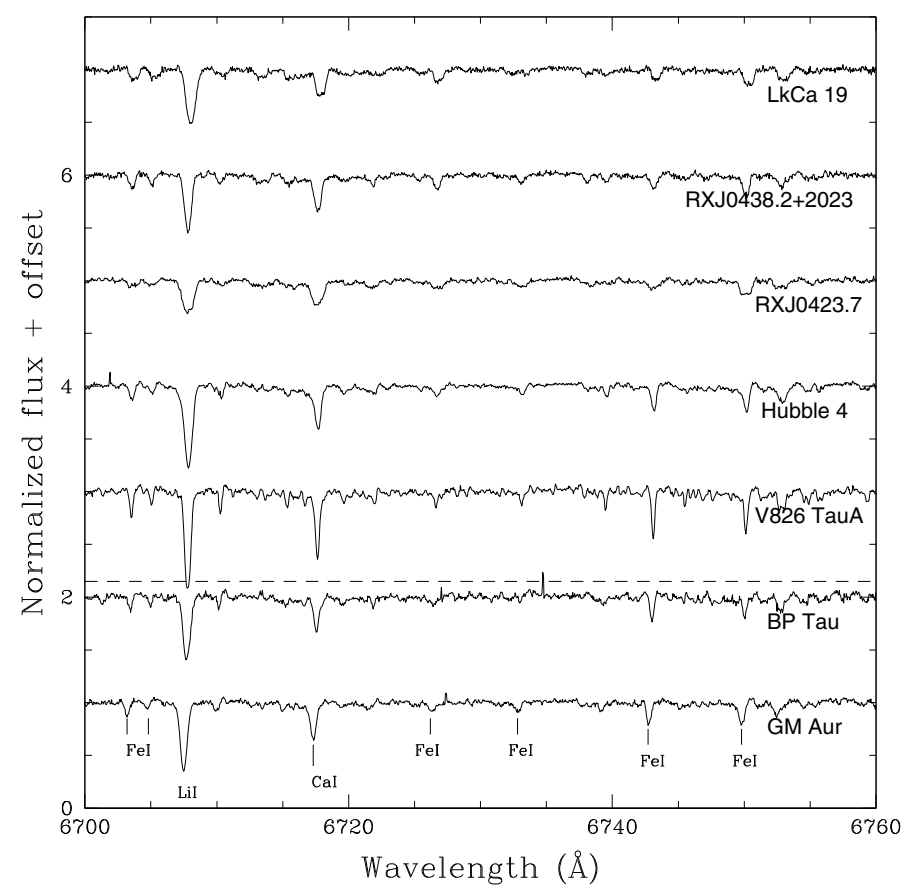

Fig. 1. Portion of the spectra of our seven sample stars between $6700 \AA$ and $6760 \AA$; the dashed line separates the WTTs from the two CTTs (GM Aur and BP Tau).

extraction, sky subtraction, and wavelength calibration. Spectra of the same star acquired in different exposures were then coadded, after checking that there were no variations in radial velocity. The spectra of seven of our sample stars are shown in Fig. 1 in the spectral window between 6700 and $6760 \AA$, where several Fe I features are present.

\section{Analysis}

\subsection{Estimate of veiling and equivalent-width correction}

Spectral veiling, which often affects spectra of pre-main sequence (PMS) stars, was estimated in the same fashion widely described in D09. Briefly, we compared the equivalent widths $(E W s)$ of the sample stars with those of stars with similar parameters belonging to the older clusters IC 2602 ( $\sim 30 \mathrm{Myr})$ and IC 2391 ( $\sim 50$ Myr): the veiling parameter is expressed as $r=$ $\left(E W_{\mathrm{IC} *} / E W_{\mathrm{TAU}}\right)-1$.

Nine spectral lines, of different atomic species, were employed in this computation, namely Ca I $5857.5 \AA$, Ca I $6102.7 \AA$, Ca I $6122.2 \AA$, Fe I $6546.3 \AA$, V I $6624.8 \AA$, Ni I $6643.6 \AA$, Fe I $6662.5 \AA$, Ca I $6717.7 \AA$, and Ti I $6743.2 \AA$. In a subsequent step, we then computed for each star the mean $r$ value coming from the different spectral lines; these average values, along with the standard deviation, are listed in Table 3. All the WTTs of our sample have negligible veiling, as expected; on the other hand, the two CTTs, GM Aur and BP Tau, exhibit quite large $r$ values. In particular, our values of $0.23( \pm 0.09)$ and $0.60( \pm 0.07)$ are in excellent agreement with the previous estimates by Basri et al. (1991), who found $0.25( \pm 0.1)$ and $0.60( \pm 0.1)$, respectively, for the two stars. Finally, the correction for spectral veiling was applied to the two "veiled" stars, by multiplying for $(1+r)$ the observed $E W$ s to obtain the correct values.
Table 2. Solar abundances derived from our analysis for $\mathrm{Fe}, \mathrm{Si}$, and $\mathrm{Ni}$.

\begin{tabular}{lcc}
\hline \hline Element & This work & AG(1989) \\
\hline $\log n(\mathrm{Si} \mathrm{I})$ & $7.54 \pm 0.03$ & 7.55 \\
$\log n(\mathrm{Fe} \mathrm{I})$ & $7.51 \pm 0.06$ & 7.52 \\
$\log n(\mathrm{Ni} \mathrm{I})$ & $6.24 \pm 0.02$ & 6.25 \\
\hline
\end{tabular}

Notes. As a comparison, we also show in Column 3 the standard abundances by Anders \& Grevesse (1989).

\subsection{Abundance analysis}

LTE abundances were derived by applying the spectral analysis program MOOG of Sneden (1973, 2002 version) and using the GAIA set of model atmospheres (Brott \& Hauschildt, priv. comm. ${ }^{1}$ ). We performed both $E W$ and spectral synthesis analyses, using the drivers abfind and synth, respectively. Radiative, Stark, and collisional broadenings were treated in the standard way, adopting for the last one the classical Unsöld (1955) approximation.

\subsubsection{Equivalent width analysis and stellar parameters}

For the five slowest rotators in our sample $\left(v \sin i \leq 18 \mathrm{~km} \mathrm{~s}^{-1}\right)$, we derived iron abundances by measuring the $E W \mathrm{~s}$. The line list was based on the one provided by Randich et al. (2006) to which we added four iron lines from the list by D09; we refer to those papers for further details on atomic parameters and, in general, for the whole procedure.

We derived solar abundances by analysing a SARG spectrum of the Sun taken with the same resolution and grating of our sample stars (see Table 2 for solar abundances of Fe, Si, and Ni). Our complete line list includes 48, 2, and 13 features of Fe I, Si I, and Ni I, respectively; however, depending on the star temperature and $S / N$, we were able to measure a different number of lines for the different stars. The final number of lines used, after the $2 \sigma$ clipping, is given in Table 3 . Assuming $T_{\text {eff } \odot}=5770 \mathrm{~K}$, $\log g_{\odot}=4.44$, and $\xi_{\odot}=1.1 \mathrm{~km} \mathrm{~s}^{-1}$ (see Randich et al. 2006), we derived $\log n(\mathrm{Fe} \mathrm{I})_{\odot}=7.51 \pm 0.04$. The $E W$ s for all the stars were measured using the IRAF task SPLOT and performing both a Gaussian fit and a direct integration to the line profile. In the case of Hubble 4, the bluer spectral region (4600-6200 A) could not be included, since this spectral window was missed because of technical problems during the observations; the analysis was thus performed using the upper (i.e., redder) orders including only 13 iron lines. Along with iron, we also derived the abundances of Si I and Ni I for the five slow rotators for which $E W$ analysis could be carried out.

Initial stellar parameters were retrieved from the XEST source catalogue. In particular, we adopted $T_{\text {eff }}$ values derived from spectral types and the calibration of KH95. After applying a $2 \sigma$ clipping to the initial line list, we derived the final $T_{\text {eff }}$ values by zeroing the slope between $\log n(\mathrm{Fe} \mathrm{I})$ and excitation potential $(\chi)$. To infer microturbulence $(\xi)$, we first assumed $1.4 \mathrm{~km} \mathrm{~s}^{-1}$ as an initial value, and then imposed the condition of there being no trends between $\log n(\mathrm{Fe})$ and $E W$ strengths. Surface gravities were computed using the expression $\log g=$ $4.44+\log \left(M / M_{\odot}\right)-\log \left(L / L_{\odot}\right)+4 \times \log T_{\text {eff }}-15.0447$, taking mass and luminosity values from summary tables by Güdel et al. (2007). We could not spectroscopically optimize these gravity

1 http://www.hs. uni-hamburg.de/EN/For/ThA/phoenix/ index.html. Biazzo et al. (2010) provide a wide discussion of why this set of model atmospheres were chosen for these young, cool stars. 
Table 3. Adopted stellar parameters, number of used $\mathrm{Fe}$ lines, and $[\mathrm{Fe} / \mathrm{H}]$ values with uncertainties.

\begin{tabular}{lcccccc}
\hline \hline Star & $r$ & $\begin{array}{c}T_{\text {eff }} \\
(\mathrm{K})\end{array}$ & $\log g$ & $\begin{array}{c}\xi \\
\left(\mathrm{km} \mathrm{s}^{-1}\right)\end{array}$ & $\mathrm{Nr}$ lines & $\begin{array}{c}{[\mathrm{Fe} / \mathrm{H}] \pm \sigma_{1} \pm \sigma_{2} \pm \sigma_{3}} \\
(E W \mathrm{~s})\end{array}$ \\
\hline GM Aur & $0.23 \pm 0.09(\mathrm{C})$ & 4100 & 3.9 & 1.7 & 17 & $0.04 \pm 0.08 \pm 0.08 \pm 0.09$ \\
BP Tau & $0.60 \pm 0.07(\mathrm{C})$ & 3900 & 3.7 & 1.3 & 18 & $-0.07 \pm 0.05 \pm 0.09 \pm 0.06$ \\
V826 TauA & $\ldots$ & 4000 & 3.8 & 1.4 & 28 & $0.00 \pm 0.09 \pm 0.08$ \\
Hubble 4 & $\ldots$ & 4000 & 3.3 & 1.5 & $13^{a}$ & $-0.08 \pm 0.05 \pm 0.08$ \\
RX J0438.2+2023 & $\ldots$ & 4850 & 4.5 & 1.9 & 27 & $0.03 \pm 0.09 \pm 0.05$ \\
\hline & & & & & $v \sin i$ & {$[\mathrm{Fe} / \mathrm{H}] \pm \sigma_{1} \pm \sigma_{2}$} \\
RX J0423.7+1537 & $\ldots$ & 4900 & 4.3 & 1.6 & $25 \pm 2$ & $0.00 \pm 0.20 \pm 0.06$ \\
AVERAGE & $\ldots$ & & & & & $-0.01 \pm 0.05$ \\
LkCa 19 & $\ldots$ & 5250 & 4.2 & 1.6 & $21 \pm 3$ & $0.20 \pm 0.20 \pm 0.06$ \\
\hline
\end{tabular}

Notes. ${ }^{(a)}$ Only the upper CCD was available

values, because of the lack of suitable Fe II lines in the spectra of the cool stars. For the warmest star within the slow-rotating sample, i.e. RX J0438.2+2023, we were able to measure only one Fe II line, lacking statistics. Therefore, photometric gravities are adopted as final values.

In Table 3, we list the final stellar parameters: the initial effective temperatures agree very well with the final ones, having a maximum difference of $70 \mathrm{~K}$ (for BP Tau). The final microturbulence values range from $1.3 \mathrm{~km} \mathrm{~s}^{-1}$ for the coolest star BP Tau to $1.9 \mathrm{~km} \mathrm{~s}^{-1}$ for the warmest one, namely RX J0438.2+2023.

\subsubsection{Spectral synthesis}

For the two WTT stars with vsini larger than $18 \mathrm{~km} \mathrm{~s}^{-1}, E W$ measurements were infeasible because of strong line blending. We thus derived a metallicity estimate using spectral synthesis method in a wavelength window of $\sim 15 \AA$, from $6700 \AA$ to $6715 \AA$, using the line list already employed in D09. We first optimised that line list by changing the $\log g f$ values, when necessary, to obtain the closest agreement between our SARG solar spectrum and the standard iron abundance by Anders \& Grevesse (1989). To compare the different observed spectra for these rapidly rotating stars with the synthetic spectra, we convolved the latter with both a Gaussian profile corresponding to our resolution of $R=29000(F W H M=0.23 \AA)$ and a rotational profile, taking into account the limb-darkening coefficients.

The following stellar parameters were adopted. Values of $T_{\text {eff }}$ and $\log g$ were retrieved from XEST; we then assumed microturbulence of $\xi=1.6 \mathrm{~km} \mathrm{~s}^{-1}$, since this is the mean value obtained from the $E W$ analysis of other sample stars. Finally, spectral synthesis allowed us to infer, as a by-product, the projected rotational velocities $v \sin i$ (Table 3).

In Figs. 2 and 3, examples of spectral synthesis are shown for the stars RX J0423.7+1537 and LkCa19, respectively. They are discussed in Sect. 4.

\subsection{Error budget}

Internal (random) errors, affecting the abundances derived by the $E W$ analysis, are due to (i) uncertainties in the oscillator strengths and $E W$ measurements, and (ii) uncertainties in the adopted set of stellar parameters. The first type of uncertainty, i.e., $\log g f$, should be minimised, given the differential nature of our analysis; on the other hand, uncertainties in EWs are clearly represented by $\sigma_{1}$ in Table 3 . Errors due to uncertainties in $T_{\text {eff }}$, $\log g$, and $\xi$ were estimated as described in D09, to which we refer for a complete and detailed description of the approach followed. In brief, we assumed typical errors of $60 \mathrm{~K}$ in $T_{\text {eff }}, 0.2$ dex in $\log g$, and $0.2 \mathrm{~km} \mathrm{~s}^{-1}$ in $\xi$ since larger errors in $T_{\text {eff }}$ and $\xi$ would introduce trends in abundances with $\chi$ and $E W$, respectively. For gravity, we instead adopted the same uncertainty as D09. We then derived the variation in the iron abundance and both $[\mathrm{Ni} / \mathrm{Fe}]$ and $[\mathrm{Si} / \mathrm{Fe}]$ by changing one stellar parameter at the time, while keeping the others unchanged, and finally we quadratically added the three contributions to obtain the total error $\left(\sigma_{2}\right)$. Furthermore, for the two "veiled" stars, we computed the abundance error due to the veiling estimate $\left(\sigma_{3}\right)$ which implied that, by assuming a conservative error equal to the rms of the mean $r$ value (see Col. 2 in Table 3), the variation in the iron abundance is \pm 0.09 and \pm 0.06 for GM Aur and BP Tau, respectively.

Uncertainties related to best-fit determination and stellar parameters affect the abundances derived from spectral synthesis: errors in the best-fit are around $0.1 \mathrm{dex}\left(\sigma_{1}\right)$. Errors in stellar parameters were computed as described by D09: a variation of $\pm 60 \mathrm{~K}$ in $T_{\text {eff }}$ and $\pm 0.2 \mathrm{~km} \mathrm{~s}^{-1}$ in $\xi$, will result in abundance changes of $\pm 0.06 \mathrm{dex}$; the effect of $\log g$ is instead smaller, reaching a maximum of $\sim 0.04 \mathrm{dex}$.

Systematic (external) errors, caused for instance by the code and/or model atmosphere, should not influence largely our final abundances, as widely described in D09.

\section{Results}

\subsection{Mean abundances}

The final abundance values are reported in Table 3, for both the $E W$ and spectral synthesis analyses. For each star (Col. 1), we list the veiling parameter $r$ in Col. 2, while the final (adopted) set of stellar parameters $\left(T_{\text {eff }}, \log g, \xi\right)$ are presented in Cols. 3-5. In Col. 6, we show the number of lines used in $E W$ analysis (upper panel) and the $v \sin i\left(\mathrm{~km} \mathrm{~s}^{-1}\right)$ as a by-product of spectral synthesis (lower panel); finally, metallicity $[\mathrm{Fe} / \mathrm{H}]$ is given in $\mathrm{Col} .7$ along with its corresponding errors.

Given the difference between the methodologies, we computed two different average values: the first technique ( $E W$ analysis) inferred $[\mathrm{Fe} / \mathrm{H}]=-0.02 \pm 0.06$, while considering the faster rotator RX J0423.7+1537 (and not considering the less secure estimate for $\mathrm{LkCa} 19$, see Sect. 4.2) we obtain a mean metallicity of $[\mathrm{Fe} / \mathrm{H}]=-0.01 \pm 0.05$. The small standard deviation from the mean, which is much smaller than expected from the 

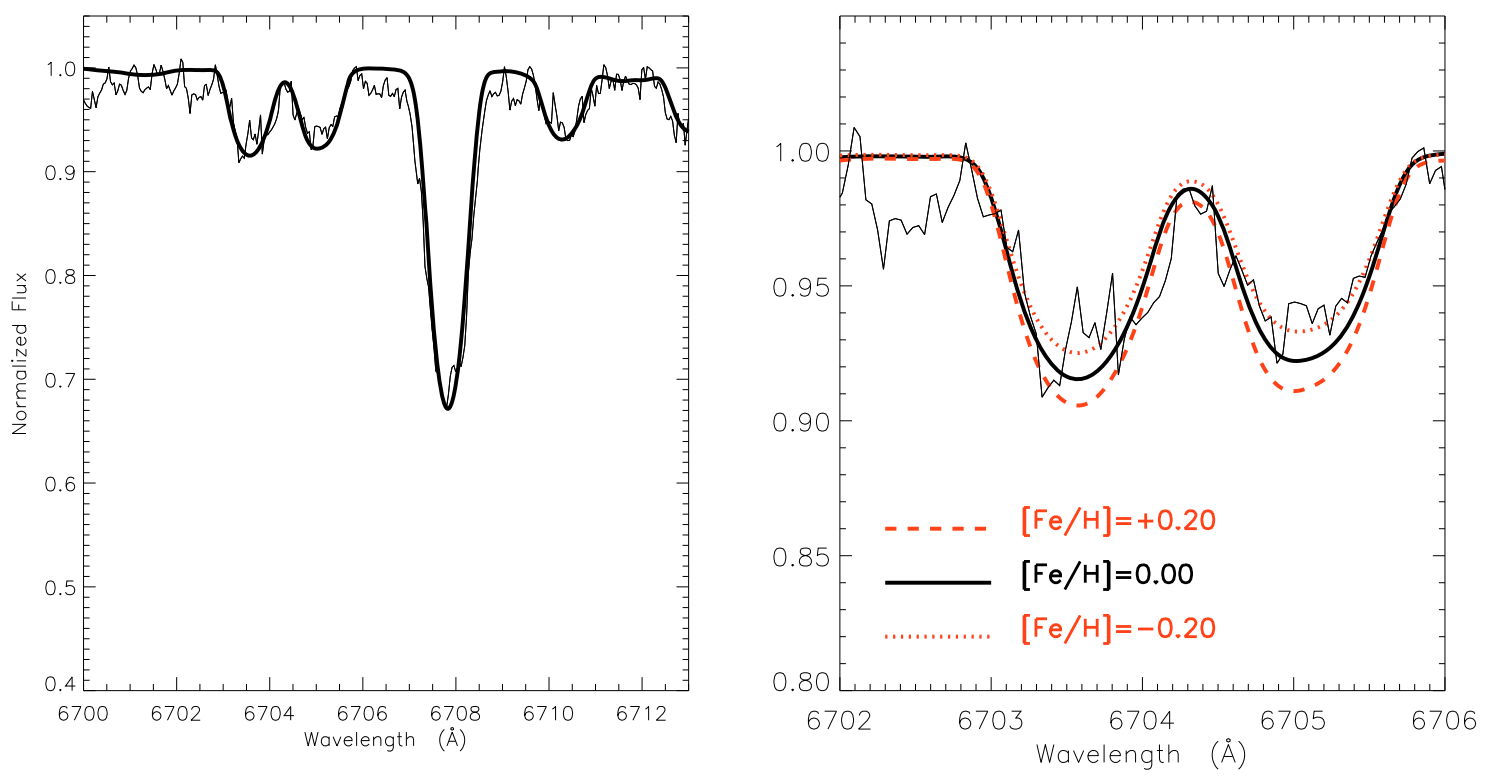

Fig. 2. Left panel: best-fit synthetic spectrum $([\mathrm{Fe} / \mathrm{H}]=0.00)$ of the star RX J0423.7+1537. Right panel: zoom on the small spectral window containing two Fe I features. Synthetic spectra obtained with the best-fit $[\mathrm{Fe} / \mathrm{H}]$ value and its $\pm 1 \sigma$ margins of error superimposed on the observed spectrum for comparison (solid, dotted, and dashed lines, respectively).
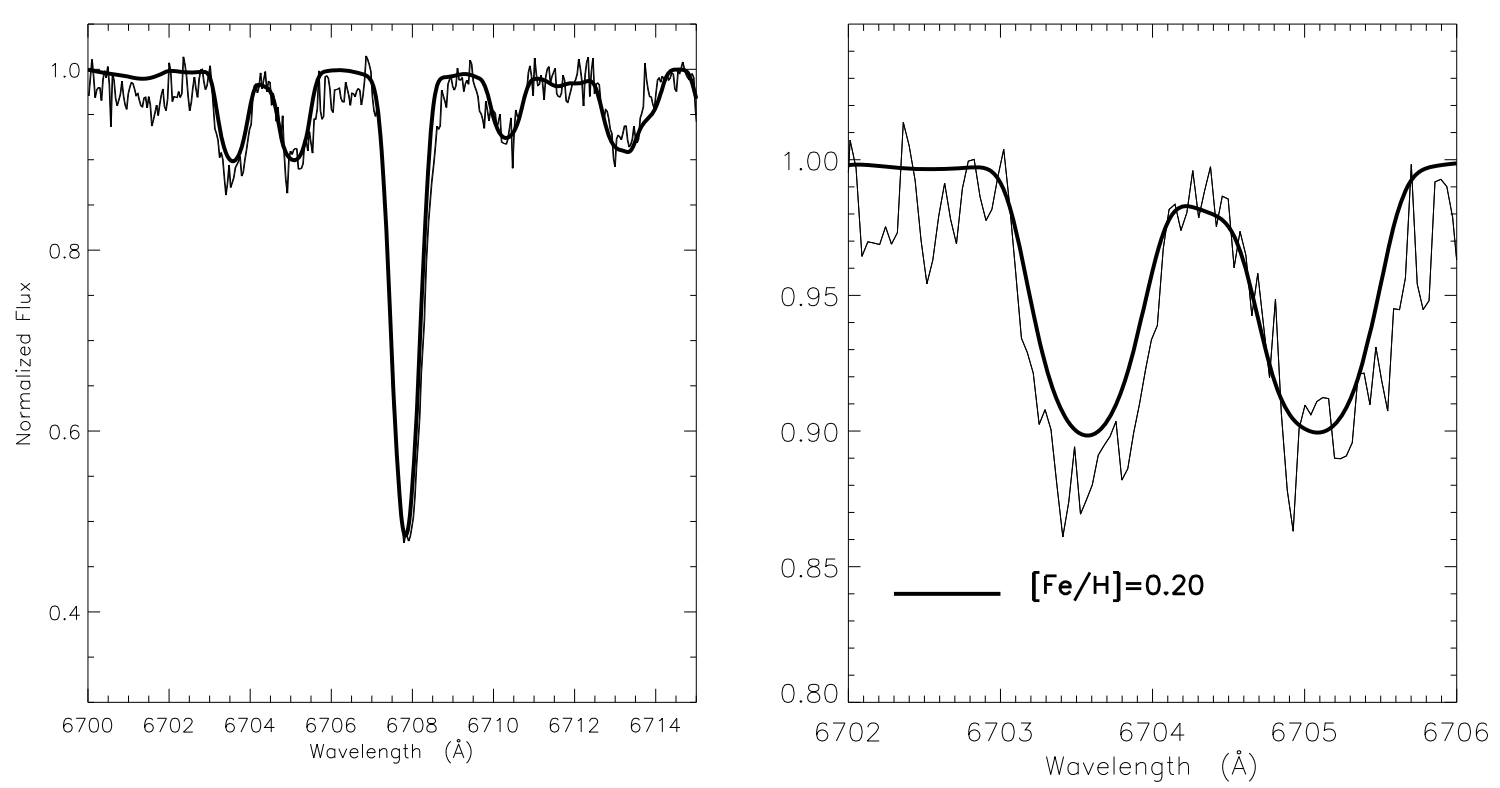

Fig. 3. Observed and synthetic spectra of the star LkCa19. The "formal" best-fit is shown in both panels as a thick solid line (see text - Sect. 4.2 for a discussion on this case).

formal errors, indicates a homogeneous solar iron abundance for Tau-Aur with no star-to-star scatter.

The $\alpha$-element $\mathrm{Si}$ I along with the Fe-peak one Ni I also confirm solar values (see Table 4 ): the average ratios $[\mathrm{Si} / \mathrm{Fe}]=$ $0.04 \pm 0.03$ and $[\mathrm{Ni} / \mathrm{Fe}]=-0.02 \pm 0.05$ indicate that Tau-Aur members are characterised by the same abundance pattern as thin disc stars of similar metallicity.

Our study provides the first abundance determination using the EWs of "deveiled" CTTs, following the work of Biazzo et al. (2010) for the Orion complex. A moderately accreting PMS star was also present in the Padgett's sample, namely CV Chamaeleonis; however, after the estimate of spectral veiling $(r=0.2 \pm 0.3)$, the $E W \mathrm{~s}$ correction was not performed and the author concluded that the derived iron abundance should be treated as a lower limit. We find that CTTs and WTTs share the same chemical composition; while this result indicates that the presence of a circumstellar disc does not affect the stellar photospheric abundances, it also strongly supports the validity of our method for estimating spectral veiling.

\subsection{Comparison with previous works}

Only one of our stars is in common with previous studies in the literature: $\mathrm{LkCa} 19$. For this star, we obtain a formal best-fit with $[\mathrm{Fe} / \mathrm{H}]=+0.2 \pm 0.2$ (see Table 3 ), suggesting a possible iron overabundance. However, the uncertainties in the stellar parameters of this star prevent us from definitively assessing its metalrich nature. As Fig. 3 shows, even the formal best-fit synthetic 
Table 4. $[\mathrm{Si} / \mathrm{Fe}]$ and $[\mathrm{Ni} / \mathrm{Fe}]$ ratios for a sub-sample of our stars.

\begin{tabular}{lcccc}
\hline \hline Star & {$[\mathrm{Si} / \mathrm{Fe}] \pm \sigma_{1} \pm \sigma_{2} \pm \sigma_{3}$} & $\mathrm{Nr}$ lines & {$[\mathrm{Ni} / \mathrm{Fe}] \pm \sigma_{1} \pm \sigma_{2} \pm \sigma_{3}$} & $\mathrm{Nr}$ lines \\
\hline GM Aur & $0.04 \pm 0.09 \pm 0.07 \pm 0.03$ & 1 & $-0.05 \pm 0.04 \pm 0.03 \pm 0.02$ & 4 \\
BP Tau & $0.08 \pm 0.07 \pm 0.08 \pm 0.02$ & 1 & $0.06 \pm 0.07 \pm 0.04 \pm 0.02$ & 5 \\
V826Tau A & $0.02 \pm 0.02 \pm 0.07$ & 1 & $0.01 \pm 0.01 \pm 0.03$ & 10 \\
Hubble4 & $\ldots$ & $\ldots$ & $-0.02 \pm 0.14 \pm 0.03$ & 2 \\
RX J0438.2+2023 & $0.03 \pm 0.07 \pm 0.06$ & 2 & $-0.06 \pm 0.04 \pm 0.03$ & 4 \\
& & & & \\
AVERAGE & $0.04 \pm 0.03$ & & & \\
\hline
\end{tabular}

spectrum cannot simultaneously reproduce both the Fe I lines at 6703 and $6705 \AA$, suggesting that the adopted $T_{\text {eff }}$, as derived from spectral-type $\left(T_{\text {eff }}=5250 \mathrm{~K}\right)$, is likely to be overestimated. We indeed note that for LkCa19 Martin et al. (1994) derived a value of $T_{\text {eff }}=4343 \mathrm{~K}$, which is probably too low. An intermediate value might be the correct one and would yield a lower $[\mathrm{Fe} / \mathrm{H}]$. A lower effective temperature would also yield a lower gravity. Since the available spectrum, and in particular the presence of several blended features, does not allow us to independently derive stellar parameters, at the present time, no final statement can be drawn about the metallicity of this star.

This star was also previously analysed by Padgett (1996), who obtained a $T_{\text {eff }}=5214 \pm 146 \mathrm{~K}$, an iron abundance $[\mathrm{Fe} / \mathrm{H}]=-0.08 \pm 0.08$, and a significantly higher microturbulence $\xi=2.8 \pm 0.5 \mathrm{~km} \mathrm{~s}^{-1}$ from an $E W$ analysis. The Padgett's line list contains about one third of spectral features with $E W \mathrm{~s}$ larger than $150 \mathrm{~m} \AA$ with a maximum value of $330 \mathrm{~m} \AA$ : these high values are probably due to blended features, given the high rotational velocity of $\sim 20 \mathrm{~km} \mathrm{~s}^{-1}$. In other words, an $E W$ analysis is really uncertain in this case, when weak metallic lines blend together and the resulting abundance probably has to be revised. To conclude, given the uncertainties in effective temperature, hence metallicity, the star LkCa19 was discarded in the computation of the average abundance for Tau-Aur, as reported in Sect. 4.1.

Focusing on the overall metallicity, in Fig. 4, our [Fe/H] determination in Tau-Aur is compared with the previous estimates by Padgett (1996) and Santos et al. (2008); their averaged values of $[\mathrm{Fe} / \mathrm{H}]=-0.01 \pm 0.13$ and $[\mathrm{Fe} / \mathrm{H}]=-0.07 \pm 0.12$, respectively, are in good agreement with our new estimate. Moreover, the $[\mathrm{Si} / \mathrm{Fe}]=0.04 \pm 0.04$ and $[\mathrm{Ni} / \mathrm{Fe}]=-0.07 \pm 0.02$ ratios derived by Santos et al. (2008) are similar to the values inferred here.

However, the figure clearly shows that the $[\mathrm{Fe} / \mathrm{H}]$ distribution from our measurements is much narrower than those obtained by both those studies. As mentioned in Sect. 1, in the work by Santos et al. (2008) the metallicity ranges from -0.18 to +0.05 , while in Padgett (1996) the internal dispersion is even larger, with $[\mathrm{Fe} / \mathrm{H}]$ ranging from -0.16 to +0.22 dex. Padgett concluded that, beyond the major uncertainties affecting young stars with respect to the older ones, "the standard deviation of $[\mathrm{Fe} / \mathrm{H}]$ within the $\mathrm{T}$ Tauri star association is larger than older clusters". However, our homogeneous abundance determination shows that, at least within our sample, the star-to-star scatter is smaller than the observational errors and that previously detected variations probably reflect these uncertainties. The same conclusion has been drawn by D09 and Biazzo et al. (2010), with both studies focusing on the abundance determination across the Orion complex. On the other hand, we note that the number of SFRs chemically analysed by considering a large sample of stars is still very limited; further investigations are necessary to confirm (or refuse) the presence of real internal scatter.

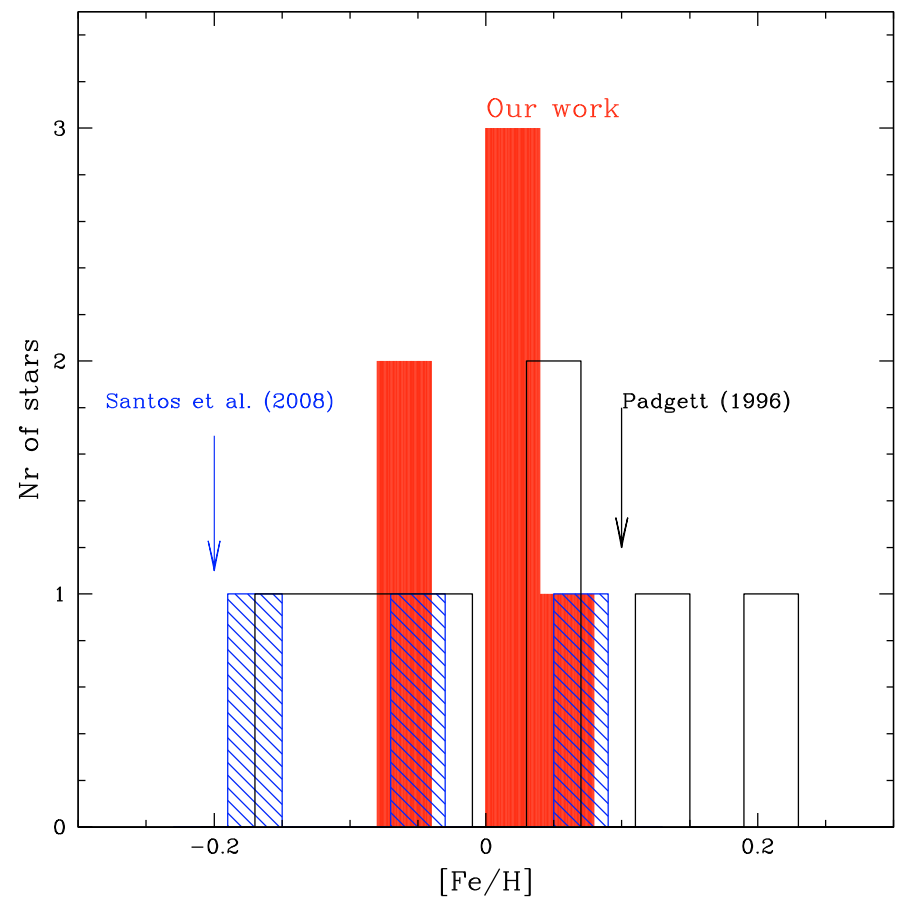

Fig. 4. Comparison of our $[\mathrm{Fe} / \mathrm{H}]$ (filled histogram) with previous estimates by Padgett (1996, empty histogram) and Santos et al. (2008, dashed histogram).

\section{Discussion}

\subsection{The metallicity of Taurus-Auriga}

The distribution of $[\mathrm{Fe} / \mathrm{H}]$ for the few SFRs and young associations with available abundance measurements was discussed by Santos et al. (2008) and Biazzo et al. (2010). None of them is more metal-rich than the Sun and, in general, they appear to be a part of a rather homogeneous sample, characterised by similar iron abundance patterns. Biazzo et al. obtained an average metallicity $[\mathrm{Fe} / \mathrm{H}]=-0.06 \pm 0.04$, considering SFRs within $500 \mathrm{pc}$ of the Sun and including the value for Tau-Aur derived in the present paper.

Nevertheless, we note that small (but larger than the errors) differences in the metallicity of the regions are present, with the Orion Nebula Cluster (ONC) and Chamaeleon being the most metal poor (with $[\mathrm{Fe} / \mathrm{H}]=-0.11 \pm 0.08$ and $-0.11 \pm 0.11$, respectively) and Tau-Aur, with a solar metallicity, being the most metal rich. Although the current sample is still too small to perform reliable statistics and draw definite conclusions, it seems that low-mass SFRs do not behave differently from high mass SFRs, that metallicity is not related to the characteristics and position of the region, and that the shape of the IMF is not related 
to the metallicity. In particular, we notice that a difference in metallicity is most likely not the reason for the different shapes of the IMF in the Chamaeleon and Taurus; indeed, as mentioned above, Chamaeleon is more metal poor than Taurus and, accordingly, should produce a smaller number of low-mass stars. We observe instead a peak at $\sim 0.8 M_{\odot}$ in the IMF of Taurus, with a deficit of lower mass stars, at variance with other regions, like Chamaeleon, showing an IMF peak at lower masses (around $0.1 M_{\odot}$, see Luhman 2004, and references therein).

\subsection{Coronal abundances}

Scelsi et al. (2007b) determined the coronal abundances of about $20 \mathrm{~T}$ Tauri stars in the Taurus Molecular Cloud. They found a clear inverse-FIP effect, with median Fe and $\mathrm{Si}$ abundances $\sim 0.2$ times the solar photospheric abundances. However, as stressed by Scelsi et al. and in Sect. 1 of this paper, the determination of photospheric abundances of young (active) stars is critically important to comparing in a homogeneous way these values with the coronal ones and drawing more definitive conclusions on possible FIP-related trends.

Three of our stars (BP Tau, V826Tau, and Hubble4) are in common with the sample of Scelsi et al. For all three stars, we obtain solar Fe and Si abundances from our analysis. This confirms a large depletion of iron and silicon in the hot coronae of these stars with respect to the photospheres, which may be attributed to condensation of iron in grains, as suggested by Scelsi et al. (2007b).

\subsection{Metallicity and planet formation}

Surveys of old solar-type stars have clearly shown that the probability of hosting giant planets increases with the metallicity of the parent star: the frequency of giant planets around stars of twice solar metallicity is about $\sim 30 \%$, to be compared with $\sim 3 \%$ for stars characterised by solar or sub-solar iron content (e.g., Santos et al. 2004; Fischer \& Valenti 2005). The origin of this correlation between metallicity and planet incidence is still debated, with the coexistence of different hypotheses, from detectability (i.e., the higher the metallicity, the greater the likelihood of planet migration and hence planet detection), to environmental effects (i.e., metals enhances the formation of dust, planetesimals, and finally cores). We mention in passing that the correlation between planet and metallicity seems to have two major caveats: (1) the different behaviours of dwarfs and giants (see Pasquini et al. 2007), giant stars hosting planets not appearing to be more metal-rich on average than star without planets; and (2) this correlation is no longer valid for $[\mathrm{Fe} / \mathrm{H}]$ values ranging from -0.7 dex to -0.3 dex (see Haywood 2009).

The high metal abundances of planet-bearing stars seem to be primordial (e.g., Pinsonneault et al. 2001; Ecuvillon et al. 2006; Gilli et al. 2006). A natural question is therefore whether young PMS stars with super-solar metallicity exist, since their circumstellar discs are commonly assumed to be the planet birthplace. As mentioned in Sect. 5.1, all the young clusters/associations for which an abundance analysis has been performed to date do not contain any high-metallicity star. For six SFRs, Santos et al. (2008) derived a slightly sub-solar metallicity, concluding that metal-rich stars in the solar proximity (and especially planet-host stars) might have formed in the inner Galaxy and then suffered radial migration across the Galactic disc. This scenario also seems to be confirmed by several studies based on both abundances and kinematics, such as Ecuvillon et al. (2007) and Haywood (2008).

Given the quite limited sample, no final conclusion can be drawn about the possible presence of metal-rich SFRs, or at least, a population of metal-rich $\mathrm{T}$ Tauri stars inside them. However, we can speculate that at the present time in the solar neighbourhood, for its young clusters/associations, the probability of forming giant planets is not as high as in the inner disc. This does not mean that no giant planets can form (and possibly survive) at all in these young stellar aggregates, but rather that the frequency of giant planets could be somewhat lower than in the inner regions of our Galaxy, where the planet-host stars are suggested to originate. To explain this, two hypotheses have been proposed: (1) the higher metal content of the inner Galaxy and thus the higher dust-to-gas ratio can enhance the probability of forming gas giant planets (for details of the core accretion model for giant planet formation see, e.g., Pollack et al. 1996; Ida \& Lin 2004; Mordasini et al. 2009a,b); (2) as an alternative, metallicity-independent approach, Haywood (2009) suggested that giant planet formation could be favoured in regions where the density of the molecular hydrogen (which is the primary constituent of these objects) is significantly higher. We note that the molecular ring of our Galaxy is located precisely at the Galactocentric radii of 3-5 kpc where these metal-rich stars are observed (Haywood 2009).

\section{Summary and conclusions}

We have presented elemental abundances of seven stars, both CTTs and WTTs, belonging to the Tau-Aur association. We have found a very homogeneous, solar metallicity for this $\mathrm{T}$ association, deriving a mean value of $[\mathrm{Fe} / \mathrm{H}]=-0.01 \pm 0.05$. Both $[\mathrm{Si} / \mathrm{Fe}]$ and $[\mathrm{Ni} / \mathrm{Fe}]$ also exhibit solar ratios and agree very well with the observed abundance pattern of thin disc stars at the same metallicity. In contrast to the previous determination of abundances in Tau-Aur, which spanned a wide range in $[\mathrm{Fe} / \mathrm{H}]$, we conclude that the internal dispersion in metallicity for this association is very similar to values derived in older open clusters. Along with our previous project in Orion and the available estimates in the literature, all the SFRs surveyed to date seem to share a similar chemical composition, suggesting a uniform ISM in the solar surroundings at the present time. In this context, we note that no metal-rich members have been detected in all the analysed young associations: this could validate the idea that metal-rich planet-host stars in the solar circle were formed in the inner disc and subsequently moved to their current location. Their not-sohigh metallicity might reflect the rather low frequency of giant planets in the solar neighbourhood, at variance with that of the inner Galactic regions. However, given that only a small number of SFRs have been chemically characterised so far, and a small number of stars per region, further investigations are mandatory to definitely help us address this controversial, but intriguing issue.

Acknowledgements. We warmly thank A. Martínez Fiorenzano for his valuable suggestions and helpful comments during the preparation of the observations. This work has made extensive use of WEBDA and SIMBAD databases. The XEST team is kindly acknowledged for having provided the full source catalogue in advance of publication. V.D. thanks Raffaele Gratton for very useful discussions on metallicity and giant planets. Misha Haywood deserves to be kindly acknowledged for sending us the material not directly available from the web. Finally, we thank the anonymous referee for her/his valuable suggestions and comments who improved the quality of our manuscript. 


\section{References}

Anders, E., \& Grevesse, N. 1989, GeCoA, 53, 197

Audard, M., Güdel, M., Sres, A., Raassen, A. J. J., \& Mewe, R. 2003, A\&A, 398, 1137

Basri, G., Martin, F., \& Bertout, C. 1991, A\&A, 252, 625

Blaauw, A. 1991, ASI Ser., 342, 125

Biazzo, K., Randich, S., \& Palla, F. 2010, A\&A, 525, A35

Briceño, C., Calvet, N., Kenyon, S., \& Hartmann, L. 1999, AJ, 118, 1354

Briceño, C., Luhman, K. L., Hartmann, L., Stauffer, J. R., \& Kirkpatrick, J. D. 2002, ApJ, 580, 317

Brinckman, A. C., Behar, E., Güdel, M., et al. 2001, A\&A, 365, 324

Da Rio, N., Gouliermis, D., \& Henning, T. 2009, ApJ, 696, 528

Davis, C. J., Scholz, P., Lucas, P., Smith, M. D., \& Adamson, A. 2008, MNRAS, 387,954

D’Orazi, V., Randich S., Flaccomio, E., et al. 2009, A\&A, 501, 973 (D09)

Ecuvillon, A., Israelian, G., Santos, N. C., Mayor, M., \& Gilli, G. 2006, A\&A, 449, 809

Ecuvillon, A., Israelian, G., Pont, F., Santos, N. C., \& Mayor, M. 2007, A\&A, 461,171

Fischer, D. A., \& Valenti, J. 2005, ApJ, 622, 1102

Gilli, G., Israelian, G., Ecuvillon, A., Santos, N. C., \& Mayor, M. 2006, A\&A, 449, 723

Güdel, M., Briggs, K. R., Arzner, K., et al. 2007, A\&A, 468, 353

Haywood, M. 2008, MNRAS, 388, 1175

Haywood, M. 2009, ApJ, 698,1

Ida, S., \& Lin, D. N. C. 2004, ApJ, 604, 388
Kenyon, S., \& Hartmann, L. 1995, ApJ, 101, 117

Kenyon, S., Dobrzycka, D., \& Hartmann, L. 1994, AJ, 108, 5

Larson, R. B. 2005 MNRAS 359, 211

Luhman, K. L. 2004, ApJ 617, 1216

Luhman, K. L., Mamajek, E. E., Allen, P. R., \& Cruz, K. L. 2009, ApJ, 703, 399

Luhman, K. L., Allen, P. R., Espaillat, C., Hartmann, L., \& Calvet, N. 2010, ApJS, 186, 111

Magazzú, A., Rebolo, R., \& Pavlenko, Ya. 1992, ApJ, 392, 152

Maggio, A., Flaccomio, E., Favata, F., et al. 2007, ApJ, 660, 1462

Martìn, E. L., Rebolo, R., Magazzú, A., \& Pavlenko, Ya. 1994, A\&A, 282, 503

Mordasini, C., Alibert, Y., \& Benz, W. 2009a, A\&A, 501, 1139

Mordasini, C., Alibert, Y., Benz, W., \& Naef, D. 2009b, A\&A, 501, 1161

Padgett, D. L. 1996, ApJ, 471, 847

Pasquini, L., Dollinger, M. P., Weiss, A., et al. 2007, A\&A, 473, 979

Pinsonneault, M. H., DePoy, D. L., \& Coffee, M. 2001, ApJ, 556, L59

Pollack, J. B., Hubickyj, O., Bodenheimer, P., et al. 1996, ICARUS, 124, 62

Randich, S., Sestito, P., Primas, F., Pallavicini, R., \& Pasquini, L. 2006, A\&A, 450, 557

Rebull, L. M., Padgett, D. L., McCabe, C.-E., et al. 2010, ApJS, 186, 259

Santos, N. C., Israelian, G., \& Mayor, M. 2004, A\&A, 415, 1153-1166

Santos, N. C., Melo, C., James, D. J., et al. 2008, A\&A, 480, 889

Sanz-Forcada, J., Favata, F., \& Micela, G. 2004, A\&A, 416, 281

Scelsi, L., Maggio, A., Micela, G., et al. 2007a, A\&A, 468, 405

Scelsi, L., Maggio, A., Micela, G., Briggs, K., \& Güdel, M. 2007b, A\&A, 473, 589

Sestito, P., Palla, F., \& Randich, S. 2008, A\&A, 487, 965

Sneden, C. A. 1973, ApJ, 184, 839

Unsöld, A. 1955, Physik der Sternatmosphären (Berlin: Springer-Verlag) 